\title{
PRIMEROS DATOS SOBRE LOS MICROMAMÍFEROS (ROEDORES E INSECTIVOROS) COETÁNEOS AL SOLUTRENSE EN LA CUEVA DE KIPUTZ IX (MUTRIKU, GUIPUZKOA, ESPAÑA)
}

\section{Preliminary data on small mammals (Rodents and Insectivores) contemporary to Solutrean age in Kiputz IX Cave (Mutriku, Gipuzkoa, Spain)}

\author{
Naroa García-Ibaibarriaga ${ }^{1}$, Xabier Murelaga ${ }^{1}$, Juan Rofes ${ }^{1}$ y Pedro Castaños ${ }^{2}$
}

Recibido el 12 de marzo de 2013. Aceptado el 11 de julio de 2013

Resumen. En este artículo describimos los micromamíferos del yacimiento paleontológico de Kiputz IX (Mutriku, Gipuzkoa, España) registrados en las tallas correspondientes a una edad arqueológica solutrense. La buena preservación de los restos óseos y la aplicación de las actuales técnicas micropaleontológicas permiten realizar la reconstrucción paleoambiental para el periodo estudiado. Los pequeños mamíferos están representados en el yacimiento por diez taxones, siete pertenecientes al Orden Rodentia (Arvicola amphibius, Microtus (Microtus) agrestis, Microtus (Microtus) arvalis, Microtus (Alexandromys) oeconomus, Chionomys nivalis, Microtus (Terricola) sp., Apodemus sylvaticus-flavicollis) y tres al Orden Eulipotyphla (Sorex) minutus, Sorex araneus-coronatus, Talpa sp.). Las variaciones ambientales determinadas a partir de la asociación de micromamíferos, sugieren que el clima en el momento de la formación del conjunto sería más frío y húmedo que en la actualidad.

Palabras clave: Solutrense, micromamiferos, paleoambiente, Kiputz, Paleolítico superior.

Abstract. In this article, the small mammal assemblage contemporary to the Solutrean age from the paleontological site of Kiputz IX cave (Mutriku, Gipuzkoa, Spain) is described. The good preservation of bones and the application of latest micropaleontological techniques allow a paleoenvironmental reconstruction of the period analyzed. Small mammals are represented in the site by ten taxa, seven belonging to the Orden Rodentia (Arvicola amphibius, Microtus (Microtus) agrestis, Microtus (Microtus) arvalis, Microtus (Alexandromys) oeconomus, Chionomys nivalis, Microtus (Terricola) sp., Apodemus sylvaticus-flavicollis) and three to the Orden Eulipotyphla Sorex minutus, Sorex araneus-coronatus, Talpa sp.). The environmental variations estimated on the basis of the micromammal association, suggested that the weather in the moment of the assemblage's formation would be colder than the one occurring in the area at the present day. The humidity also could be higher than the current one.

Keywords: Solutrean, small mammals, paleoenvironment, Kiputz, Upper Paleolithic.

(') Departamento de Estratigrafía y Paleontología. Facultad de Ciencia y Tecnología. Universidad del Pais Vasco UPV/EHU. Barrio Sarriena s/n. E-48940 Leioa (Bizkaia, España) naroa.garcia@ehu.es; xabier.murelaga@ehu.es; juan.rofes@ehu.es

(2) Sociedad de Ciencias Aranzadi, Geo-0. Santimamiñe 41. Leioa (Bizkaia, España).pedrocastanos@yahoo.es 


\section{INTRODUCCIÓN}

Las asociaciones a lo largo de la secuencia estratigráfica de micromamíferos presentes en el registro fósil de un yacimiento, tanto arqueológico, como paleontológico, están estrechamente ligadas a las características ambientales del entorno. Por esta razón, cualquier alteración que se de en estas, generará variaciones considerables que afectarán al conjunto de pequeños mamíferos. Esto hace que los micromamíferos sean de gran utilidad a la hora de realizar reconstrucciones ambientales y climáticas. Además, el hecho de que la mayoría de estos taxones sigan actualmente estando presentes en la cornisa cantábrica, hace posible un acercamiento a sus afinidades ecológicas desde un punto de vista actualista (Cuenca-Bescós et al. 2008; Pemán 1985; Sesé 2005).

En este artículo se recogen los primeros datos sobre los micromamíferos de la secuencia fosilifera coetánea al Solutrense del yacimiento de Kiputz IX.

\section{KIPUTZ IX}

El yacimiento de Kiputz IX está ubicado al Este del Monte Arno (617 m), en el municipio de Mutriku (Gipuzkoa), a $119 \mathrm{~m}$ sobre el nivel del mar (s.n.m.), al noreste de las cavidades de Kiputz I y Kiputz II (Fig. 1). Es un entorno geológico de calizas urgonianas del Cretácico inferior, en el que los principales collados y montes son Kalbario (296 m),

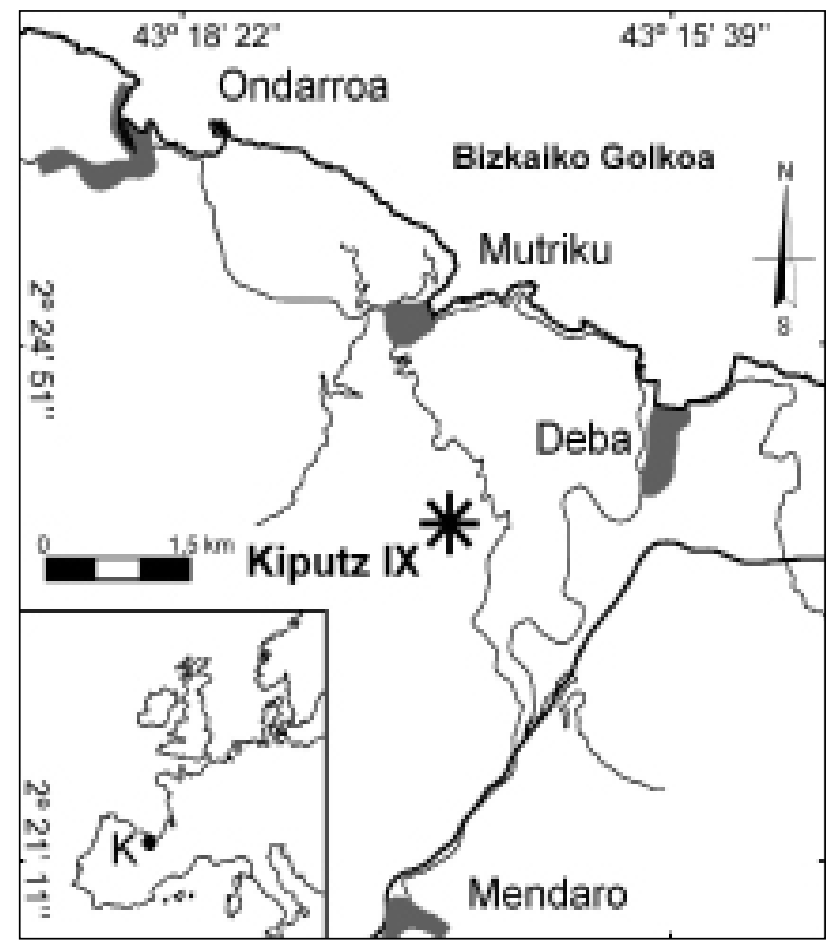

A FIgURA 1. Localización geográfica del yacimiento de Kiputz IX (Mutriku, Gipuzkoa, España).
Kanpantzar (628 m), Presoleta (537 m) y Jaungoikomendi (532 $\mathrm{m}$ ) entre otros. En esta zona geográfica se da una alta densidad de evidencias arqueológicas, con yacimientos tan característicos como Ermittia, Praile Aitz I o Zerratu.

Fue descubierto en el año 2002 como resultado de las prospecciones sistemáticas del grupo Munibe de Azkoitia. Tras realizar una cata de un metro cuadrado en marzo del 2003, las excavaciones sistemáticas fueron acometidas entre 2004 y 2007, bajo la dirección de Pedro M. ${ }^{\text {a Castaños }}$ (Castaños 2005, 2006, 2007 y 2008).

La importancia del yacimiento de Kiputz estriba en la cantidad de restos en buen estado de conservación que ha proporcionado. El origen de la formación del yacimiento es el de una trampa en la que caían los animales (sobre todo herbívoros), cuyos restos se iban acumulando en el fondo de una sima. La acumulación de macromamíferos no ha sido producida ni por humanos ni por depredadores, lo cual explica la integridad de los restos encontrados. En el País Vasco los yacimientos paleontológicos son muy escasos, aunque se han constatado recogidas puntuales en superficie de osamentas halladas en cavidades cársticas como en Pagolusieta (Altuna 1980), Legintxiki (Castaños 1996) o Illobi (Villaluenga 2011). En cambio Kiputz ha sido excavado sistemáticamente y con una metodología arqueo-paleontológica (el sedimento se ha extraído siguiendo el sistema de tallas artificiales de unos 5-10 cm adaptadas a los niveles estratigráficos naturales).

Por otra parte, es un enclave de primer orden al proporcionar una importante muestra de restos de macromamíferos, como son los conjuntos más importantes de reno (23 individuos) (Castaños et al. en prensa) y de bisonte (18 individuos) (Castaños et al. 2012) de toda la Península lbérica. Entre otros destaca un cráneo prácticamente completo de Bison priscus o bisonte estepario, pieza única en toda la Península lbérica.

La cavidad presenta una disposición en forma de jarra y un relleno de 4,2 $\mathrm{m}$ de potencia, donde se han diferenciado 8 niveles ( $\mathrm{A}-\mathrm{H} ;$ Fig. 2). Existen diversas dataciones de carbono 14 convencional, la más reciente del nivel C (13424-13766 cal BP), mientras que en el nivel basal se ha obtenido una fecha de 36625-38560 cal BP. Existen dos dataciones coetáneas al periodo cultural arqueológico denominado Solutrenses en el nivel F, una de 21369-18140 \pm 90 BP (talla 34) y otra de $19870 \pm 150$ BP (talla 39).

Es posible encontrar dataciones similares a la de la talla 39 en La Riera (nivel X 24672-22530 cal BP, $19820 \pm 390$ BP) (Straus y Clarck, 1986) o Las Caldas (nivel XVI 24133-22426 cal BP, $19510 \pm 330$ BP) (Corchón 1990). Por su parte, tanto Las Caldas, como Aitzbitarte IV (Altuna, 1972), tienen fechas similares a la de la talla 34, con una datación de 2250221068 cal BP el primero (nivel VII $18250 \pm 300$ BP) y $21806-$ 21077 cal BP el segundo (nivel VII $17950 \pm 100$ BP).

Por lo tanto, la secuencia estudiada en este trabajo se sitúa en el OIS 2 o Último Máximo Glacial, el cual se carac- 
teriza por ser una fase fría y árida con pulsaciones de mayor humedad y no tan frías. Sin embargo, en nuestras latitudes este periodo no se debió de corresponder con las condiciones climáticas más extremas que sí se dieron en otras zonas del continente europeo, como se desprende de estudios realizados a partir de los pequeños vertebrados (López-García et al. 2013).

\section{METODOLOGÍA}

El material referido en este artículo pertenece al cuadro $3 C$ de las tallas 35, 36, 37 y 39 del nivel F (Fig. 2), el cual tiene una potencia de unos $50 \mathrm{~cm}$ y está compuesto por arcillas marrones, alguna brecha caliza y abundantes restos de macrovertebrados. Fue excavado en la campaña del 2004, recogiéndose diversas muestras de sedimento para su posterior análisis microfaunístico. Antes de comenzar el proceso de lavado, la cantidad de sedimento de cada capa

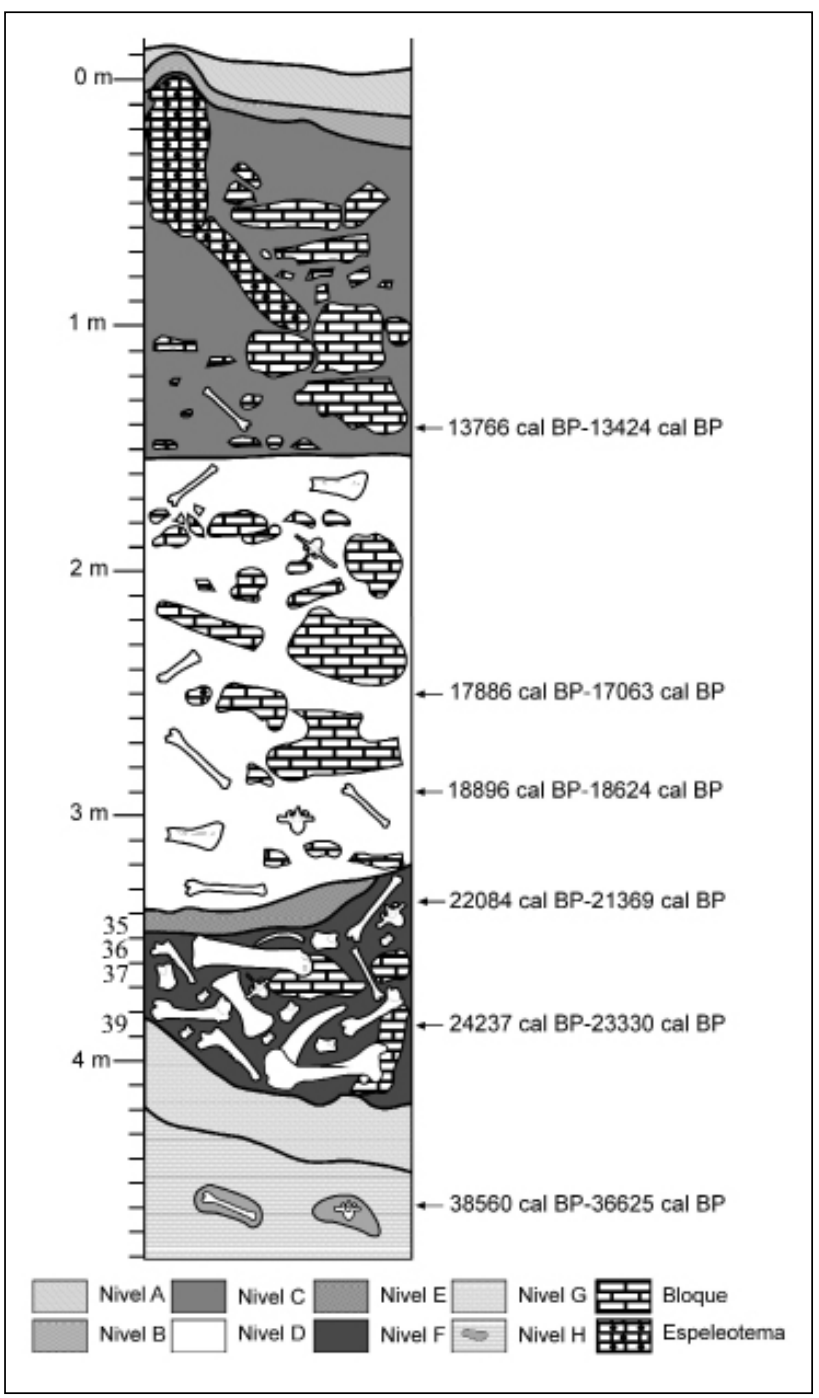

\ Figura 2. Columna estratigráfica del yacimiento de Kiputz IX (Mutriku, Gipuzkoa, España). fue calculada en kilos para luego poder identificar la riqueza en restos fósiles por muestra. De esta forma se calculó que las tallas analizadas en este estudio comprendian un total de $36 \mathrm{~kg}$ antes de su lavado, el cual se llevó a cabo utilizando como tamiz inferior el de $0.5 \mathrm{~mm}$ de luz de malla.

El residuo resultante del lavado del sedimento con agua, libre de arcillas y limos, fue separado en dos fracciones de distinto tamaño ( $>2 \mathrm{~mm}$ y 2-0.5 $\mathrm{mm}$ ) para facilitar el triado de los restos fósiles. La fracción gruesa no necesitó de ningún dispositivo óptico para su procesado, mientras que la fracción menor fue triada con una lupa estereoscópica modelo Nikon Smz 1500 10x. Una vez agrupados los restos fósiles según criterios anatómicos, para la determinación taxonómica se han tenido en cuenta el primer molar inferior o $\mathrm{m} 1$ en los Arvicolinae, el molar más representado en el género Apodemus y el elemento anatómico mejor representado en el caso de los insectívoros.

Los molares de los roedores e insectívoros han sido fotografiados empleando un equipo Nikon compuesto por una cámara de fotomicrografía (DS-5m) y una unidad de control y monitorización (DS-L1) acoplados a la lupa citada anteriormente.

La nomenclatura sistemática sigue los trabajos de Wilson y Reeder (2005). En los arvicólidos y múridos la nomenclatura de la dentición (Fig. 3) está basada en Van der Meulen (1973) y Heinrich (1978), mientras que para los sorícidos se ha utilizado el trabajo de Reumer (1984).

Las mediciones han sido necesarias a la hora de establecer la taxonomía especifica del genero Arvicola, para lo cual nos hemos basado en el índice SDO de Heinrich (1982).

Contabilizando el elemento anatómico más representado según la posición del elemento en el esqueleto (derecho o izquierdo) se ha especificado el Número Mínimo de Individuos (NMI).

Las dataciones presentadas en este estudio están calibradas con el programa/software 0xCal 4.1.7. (Bronk Ramsey 2009), basado en la curva de Calibración INTCAL09 (Reimer et al. 2009).

\section{ESTUDIO SISTEMÁTICO (LAM. 1 Y TAB. 1)}

Orden RODENTIA Bodwich, 1821

Familia Cricetidae Fischer, 1817

Género Chionomys Miller, 1908

Chionomys nivalis Martins, 1842. Neverón o Topillo nival. Lam. 1.1

\section{Material}

Esta especie se encuentra representada en Kiputz IX por un $\mathrm{m} 1$ encontrado en el talla 36. 


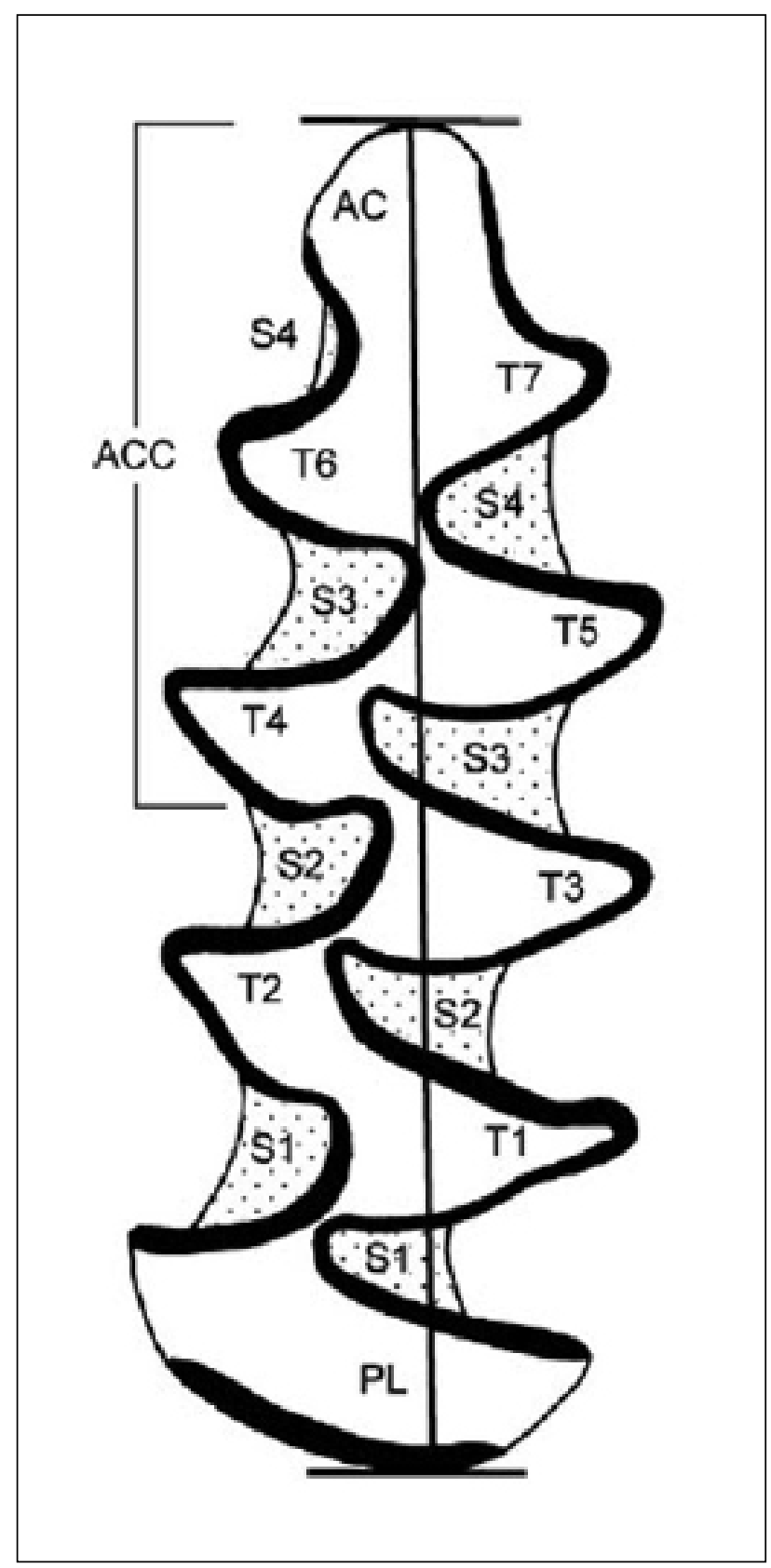

A Figura 3. Nomenclatura utilizada en la descripción de microtidos y arvicolidos, según Meulen (1973) y modificada por Kalthoff et al. (2007).

Discusión y conclusiones

El Topillo nival es el topillo "tipo" Microtus más grande de la Península Ibérica. Su lóbulo anterior es característico al presentar forma de seta, con el sombrero fuertemente inclinado hacia el lado labial.

Actualmente vive en las zonas montañosas, aunque también se constata su presencia en zonas rocosas a cotas bajas, casi al nivel del mar. Esta adaptado a las zonas abiertas de escasa cobertura vegetal y terrenos rocosos con fisuras o pedreras, siendo justamente la disponibilidad de terrenos rocosos lo que condiciona su presencia.
Género Microtus Schrank 1798

Subgénero Microtus (Terricola) Fatio, 1867

Microtus (Terricola) sp. Lam. 1.2-3

Material

En total, se han identificado ocho $\mathrm{m} 1$ pertenecientes a este subgénero en todas las tallas, a excepción de la 37.

Discusión y conclusiones

Los molares pertenecientes a esta especie son hypsodontos y arrizodontos. Los $\mathrm{m} 1$ se caracterizan por tener el T4 y T5 confluentes, formando el llamado rombo pitimiano. El cuello del bucle anterior es ancho y se une a los triángulos T6 y T7, que también son confluentes entre sí.

Para poder diferenciar las especies pertenecientes al subgénero Microtus (Terricola) sp., es necesario disponer de un material amplio, que al no ser nuestro caso, hace que la clasificación no alcance la determinación de especie. En el Cantábrico coexisten las especies Terricola lusitanicus y Terricola pyrenaicus. Los miembros del género Terricola son especies cavadoras y propias de praderas húmedas con suelos profundos y abundante cobertura herbácea.

Subgénero Microtus (Microtus) Schrank 1798

Microtus (Microtus) agrestis (Linnaeus, 1761). Topillo agreste. Lam. 1.4-5

Material

La determinación de esta especie se ha basado en el estudio de los primeros molares inferiores. En las tallas analizadas han aparecido un total de 10 de estas piezas dentarias. No está presente en el talla 39 , mientras que en el talla 36 hay ocho $\mathrm{m} 1$.

\section{Discusión y conclusiones}

La dentición de Microtus (Microtus) agrestis es de pequeña talla y arrizodonta. El m1 presenta tres triángulos cerrados en el complejo posterior y cuatro cerrados o escasamente confluentes en el anterior. A diferencia del T6 y el T7, el T4 y el T5 no son ni confluentes y ni simétricos. El esmalte en los triángulos es más grueso en la cara mesial, presentando una marcada alternancia de los ángulos entrantes. En cuanto a los ángulos, estos terminan en punta y son curvados hacia la cara anterior del diente. Presenta grandes similitudes con Microtus (Microtus) arvalis, lo cual muchas veces dificulta su identificación.

En cuanto al tipo de hábitat, el topillo agreste vive en una gran variedad de medios, desde zonas boscosas a zonas 


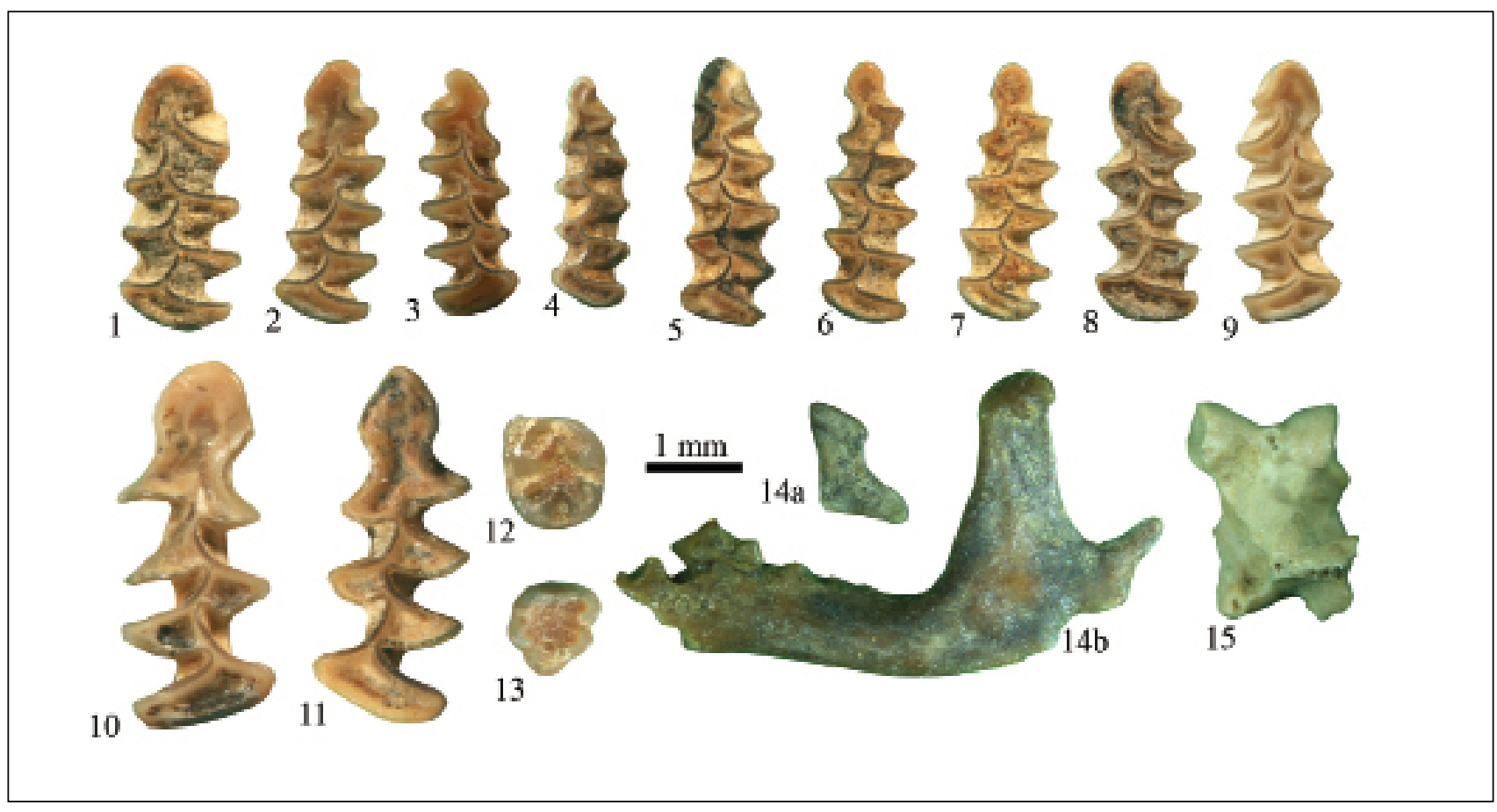

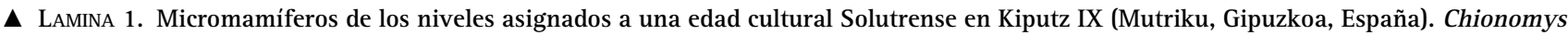
nivalis 1. $\mathrm{m} 1$ izquierdo, vista oclusal; Microtus (Terricola) sp. $2 . \mathrm{m} 1$ izquierdo, vista oclusal; 3 . $\mathrm{m} 1 \mathrm{derecho,} \mathrm{vista} \mathrm{oclu-}$ sal; Microtus (Microtus) agrestis $4 . \mathrm{m} 1$ izquierdo, vista oclusal; 5. m1 izquierdo, vista oclusal; Microtus (Microtus) arvalis 6. $\mathrm{m} 1$ izquierdo, vista oclusal; 7. $\mathrm{m} 1$ izquierdo, vista oclusal; Microtus (Alexandromys) oeconomus 8. m1 izquierdo, vista oclusal; 9. $\mathrm{m} 1$ derecho, vista oclusal; Arvicola amphibius 10. $\mathrm{m} 1$ derecho, vista oclusal; $11 . \mathrm{m} 1$ izquierdo, vista oclusal; Apodemus gr. sylvaticus-flavicollis 12. m2 izquierdo, vista oclusal; 13. M2 izquierdo, vista oclusal; Sorex gr. araneus-coronatus 14a. cóndilo mandibular, vista posterior; 14b. mandíbula izquierda, vista lateral; Talpa sp. 15. falange I, vista ventral.

abiertas con cierta cobertura vegetal. Las condiciones ideales son zonas con una cobertura vegetal espesa. Su distribución actual parece restringirse al tercio norte peninsular.

Microtus (Microtus) arvalis (Pallas, 1778). Topillo campesino. Lam. 1.6-7

\section{Material}

Es el roedor mejor representado en el yacimiento, con 27 molares. Está presente en todas las tallas, aunque no con la misma frecuencia.

\section{Discusión y conclusiones}

Como ya se ha señalado anteriormente, esta especie es semejante a Microtus (Microtus) agrestis. La diferencia reside en que el $\mathrm{T} 4$ y $\mathrm{T} 5$ y sobre todo el T6 y $\mathrm{T} 7 \mathrm{del} \mathrm{m} 1$ de esta especie presentan una disposición casi simétrica y en paralelo, además de que el complejo anterior es mucho más redondeado.

Es una especie más oportunista que su congénere, por lo que su repartición es más extensa y menos discontinua, extendiéndose hacia el sur en la Península lbérica. Aun cuando requiere de cierta humedad, puede encontrarse también en

\begin{tabular}{|c|c|c|c|c|c|c|c|c|c|c|c|}
\hline Talla & 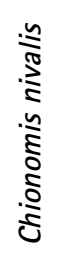 & 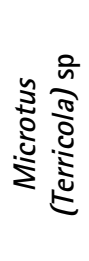 & 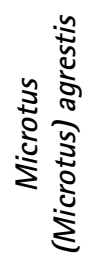 & 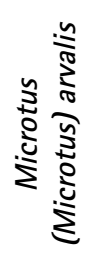 & 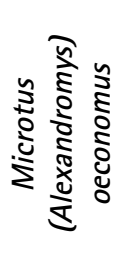 & 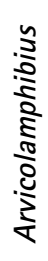 & 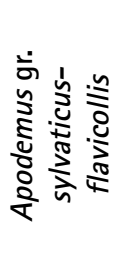 & 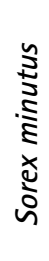 & 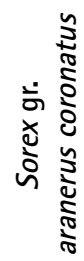 & $\begin{array}{l}\dot{0} \\
\frac{2}{\pi} \\
\frac{2}{\pi}\end{array}$ & $\begin{array}{l}\bar{\pi} \\
\text { 苛 }\end{array}$ \\
\hline 35 & 0 & 3 & 1 & 9 & 9 & 0 & 1 & 1 & 16 & 0 & 40 \\
\hline 36 & 1 & 2 & 8 & 13 & 9 & 1 & 1 & 0 & 13 & 0 & 48 \\
\hline 37 & 0 & 0 & 1 & 4 & 2 & 0 & 0 & 0 & 3 & 0 & 10 \\
\hline 39 & 0 & 3 & 0 & 1 & 5 & 1 & 1 & 0 & 4 & 1 & 16 \\
\hline
\end{tabular}

\ Tabla 1. Distribución del Número Mínimo de Individuos (NMI) de roedores e insectívoros de las tallas 35, 36,37 y 39 de Kiputz IX (Mutriku, Gipuzkoa, España). 
zonas abiertas con abundante vegetación herbácea o arbustiva, coincidiendo su distribución con el piso bioclimático supramediterráneo (inviernos largos y fríos y precipitaciones anuales entre 600 y $1200 \mathrm{~mm}$ ). A pesar de ello, al ser una especie cavadora, su presencia está más limitada por las características del suelo que por las condiciones climáticas.

\section{Subgénero Microtus (Alexandromys) Ognev, 1914}

Microtus (Alexandromys) oeconomus (Pallas, 1776). Topillo nórdico. Lam. 1.8-9

Material

Los $25 \mathrm{~m} 1$ identificados como pertenecientes a esta especie están distribuidos por las cuatro tallas analizadas, aunque de forma heterogénea.

Discusión y conclusiones

Los primeros molares inferiores de esta especie se caracterizan por tener dos anticlinales en la cara medial y mesial. El T6 está apenas marcado, por lo que el lóbulo anterior es asimétrico. También presenta tres triángulos cerrados en el lado labial y cinco en el lingual, con T4 y T5 alternos.

Aun cuando actualmente el topillo nórdico es un habitante de tundra y taiga holártica, también se ha registrado su presencia, aunque en proporciones pequeñas, en los niveles menos fríos del Pleistoceno y del Holoceno en Amalda (Pemán 1989) o El Mirón (Cuenca-Bescós y García-Pimienta 2012), en épocas en las que ya había desaparecido en otras zonas de Europa. Se considera como indicadora de hábitats acuáticos y semi-acuáticos.

Género Arvicola Lacèpéde, 1799

Arvicola amphibius (Linnaeus, 1758). Rata topera. Lam. 1.10-11

Material

Se han recuperado dos molares de la especie Arvicola amphibius. Esta especie se encuentra únicamente en las taIlas 36 y 39.

\section{Discusión y conclusiones}

La determinación sistemática de los arvicolinos se establece a partir de la morfología y biometría de los dientes yugales o molares, que suelen ser de gran talla y arrizodontos. El primer molar inferior presenta una morfología característica en el complejo anterocónido, con el bucle anterior y los triángulos T4 y T5 confluentes. Por su parte, el complejo trigónido-talónido está formado por tres triángulos cerrados. Puesto que el grosor del esmalte que rodea la dentina de los triángulos es mayor en la cara mesial que en la distal (Heinrich 1982), es posible determinar que el arvicólido presente en Kiputz IX es Arvicola amphibius.

En la actualidad, la rata topera solo se encuentra en el norte peninsular. Aunque habita en medios fluviales lacustres, no es tan ribereño como su congénere Arvicola sapidus. Son sus hábitos cavadores los que limitan su distribución, ya que precisa de suelos relativamente profundos y húmedos. Es por ello por lo que prefiere espacios abiertos como praderas húmedas y no bosques densos.

Familia Muridae Illiger, 1811

Género Apodemus Kaup, 1829

Apodemus sylvaticus (Linnaeus, 1758) - Apodemus flavicollis (Melchior, 1834). Ratón de campo, ratón leonado. Lam. 1.12-13

Material

Exceptuando en la talla 37, esta especie es constante en todas las tallas, aun cuando su representación es más bien baja (un mínimo de un individuo por talla).

Discusión y conclusiones

Debido a la gran similitud morfológica de los dientes de las diferentes especies de talla media del género Apodemus, su discriminación es complicada. Los $\mathrm{m} 1$ se caracterizan por tener 4 raices principales y la superficie oclusal con 6 cúspides principales. Adicionalmente, entre el T2 y T3 existe una cúspide y las cúspides del T4 y T7 no están conectadas.

Son especies que se pueden encontrar tanto en espacios con cobertura vegetal densa, arbórea y arbustiva, como en descubiertos, aunque cuando su proporción aumenta en las muestras esto suele ser indicativo de medios más boscosos y más cálidos (Cuenca-Bescós et al. 2009).

Orden EULIPOTYPHLA Waddell, Okada y Hasegawa, 1999

Familia Soricidae Fischer, 1814

Género Sorex (Linnaeus, 1758)

Sorex minutus Linnaeus, 1766. Musaraña enana

Material

La presencia de este pequeño insectivoro solo se ha constatado en la talla 35.

\section{Discusión y conclusiones}

La mandibula asignada a esta especie presenta los caracteres propios del género, como la fosa interna grande y triangular, cóndilo mandibular con facetas moderadamente 
separadas y con área interarticular relativamente amplia, incisivo superior fisidencia o no y con M1 y M2 con crestas entocónidas (Reumer, 1984). Dentro del género Sorex, $S$. minutus es una de las musarañas de dientes rojos más pequeñas, aunque en este caso la coloración ha desaparecido completamente de la dentición.

Es una especie que se conoce en Europa desde el Pleistoceno Inferior. Vive en ambientes húmedos de pluviosidad superior a los 600-700 mm anuales, desde el nivel del mar hasta los $2000 \mathrm{~m}$ de altitud. Necesita de una densa cobertura vegetal y de humedad ambiental.

Sorex araneus Linnaeus, 1758 - Sorex (Sorex) coronatus Millet, 1828. Musaraña bicolor-musaraña tricolor. Lam. 1.14a-14b

Material

Es la especie de micromamífero mayoritario en el yacimiento, con al menos 36 individuos repartidos de forma desigual por los cuatro niveles estudiados.

\section{Discusión y conclusiones}

Las características principales de este grupo son las descritas en Sorex minutus, de la cual se diferencian por su mayor tamaño. Aun así, distinguir Sorex aranerus de Sorex coronatus es difícil basándonos en elementos craneales, por lo que se opta por agruparlas.

En la actualidad, conviven tres especies de Sorex al norte de la Península Ibérica: Sorex araneus, Sorex coronatus y Sorex granarius, con una amplia distribución en el norte peninsular. Están ligadas a ambientes húmedos con buena cobertura vegetal, sobre todo bosques caducifolios y riberas (Pokines 1998; Cuenca-Bescós et al. 2008, 2009).

Familia Talpidae Fischer, 1814

Género Talpa Linnaeus, 1758

Talpa sp. Lam. 1.15

\section{Material}

Los topos están representados en este yacimiento por un único individuo identificado en el talla 39 mediante una primera falange.

\section{Discusión y conclusiones}

Los topos, aunque son especies grandes y con un esqueleto determinable prácticamente en su totalidad, están presentes en los yacimientos arqueológicos y paleontológicos, si bien de forma reducida al ser presas difíciles incluso para los depredadores más frecuentes. Al no contar en la literatura científica con suficiente caracteres de diferenciación, hemos optado por dejar la identificación en el género.

Actualmente cohabitan dos especies de tálpidos en la cornisa cantábrica: Talpa europea y Talpa occidentalis. Talpa es un género euritermo asociado a lugares húmedos de suelos profundos y con buena cobertura vegetal, arbustiva o arbórea.

\section{INTERPRETACIÓN PALEOECOLÓGICA Y PALEOCLIMÁTICA}

\subsection{Clima y vegetación actual en el entorno de la cueva de Kiputz IX}

La zona litoral de Mutriku presenta una temperatura media anual de unos $13,4^{\circ} \mathrm{C}$. Altitudinalmente se encuentra por encima de los $119 \mathrm{~m}$ s.n.m., correspondiendo por lo tanto al piso bioclimático colino. El régimen de precipitaciones es el de un clima oceánico, con lluvias presentes todo el año y con inviernos suaves y veranos frescos.

Actualmente el paisaje vegetal es frondoso, con un manto de encinas cantábricas (Quercus ilex) que constituyen una de las mayores masas forestales de esta especie en el norte de la Península lbérica (Edeso 1993).

\subsection{Interpretación paleoecológica y paleoclimática}

Los taxones presentes en Kiputz IX perviven en la actualidad, aunque no todos se hallan entre la fauna actual de la cornisa cantábrica. Por este motivo, siguiendo la perspectiva del Actualismo, podemos estimar el tipo de hábitat y las afinidades ecológicas de cada uno de los taxones estudiados. Hemos asignado la fauna de Kiputz IX a 5 tipos de hábitat empleando los trabajos de Blanco (1998), Cuenca-Bescós et al. (2008), Kalthoff et al. (2007), López-García et al. (2011), Palomo y Gisbert (2002), Pemán (1985) y Sesé (2005).

Este tipo de clasificaciones presentan ciertos límites, ya que es necesario tener en cuenta que las transiciones entre biotopos son graduales, y que existen especies capaces de adaptarse a más de un tipo de hábitat, o que este cambie según la región. También cabe la posibilidad de que las especies fósiles no tuviesen los mismos requerimientos ecológicos que sus representantes actuales.

Los pequeños mamíferos presentes en Kiputz IX se agrupan en cinco hábitats según sus preferencias ecológicas:

- Ribera: masas de agua como arroyos, lagos y estanques o marismas. Microtus (Alexandromys) oeconomus.

- Pradera húmeda: pradera de hoja perenne y suelos profundos con mucho pasto y capa vegetal. Es indicativo de condiciones húmedas. Arvicola amphibius, Microtus (Alexandromys) oeconomus, Microtus 
(Terricola) sp., Sorex minutus, Sorex gr. araneus-coronatusy Talpa sp.

- Pradera: zonas abiertas y herbazales con densa cobertura herbácea o arbustiva aunque con cierta humedad ambiental. Microtus (Microtus) arvalis y Microtus (Microtus) agrestis.

- Montaña: zonas montañosas abiertas con prados secos y sustrato rocoso. Indicativo de condiciones climáticas esteparias. Chionomys nivalis

- Bosque y matorral: bosque maduro con abundante cobertura arbustiva o arbórea, incluyendo límites de bosques. Indicativo de condiciones climáticas cálidas. Apodemus sylvaticus-flavicollis.

Las especies Arvicola amphibius, Microtus (Microtus) arvalis y Chionomys nivalis no se encuentran actualmente en el entorno del yacimiento, aunque si en otras zonas de la región cantábrica. Por su parte, Microtus (Alexandromys) oeconomus desapareció de la región cantábrica probablemente a comienzos del Holoceno.

\subsection{Tafonomía}

Al igual que la mayoría de los casos, la acumulación de micro restos de Kiputz IX está producida mayormente por la acción de depredadores como rapaces y/o pequeños mamíferos. Los restos asignados a roedores no presentan prácticamente marcas de corrosión producidas por jugos gástricos.
Y los que hay son moderados, por lo que el agente productor de la tanatocenosis primaria debió de ser algún depredador incluido en la categoria 1 de Andrews (1990), posiblemente alguna rapaz nocturna. En lo que respecta a los Sorícidos, es llamativa la digestión que presentan algunos restos, ya que como se ha comentado anteriormente, algunas piezas dentarias incluso han perdido el pigmento rojo-amarillento que tiñe la parte apical de los dientes (en estos casos el rastro solo es visible bajo luz ultravioleta, como ya ha sido constatado en otros yacimientos: Rofes et al. 2012). Por lo tanto, podríamos pensar que en este caso el autor se incluiría en la categoría 2 o 3 de Andrews (1990).

Aunque la tafocenosis está formada por una alta proporción de incisivos y molares sueltos con respecto a mandíbulas y maxilares, esta relación es similar a la que suelen presentar aquellos yacimientos en los que los restos han sufrido pequeñas alteraciones antes de ser enterrados. Lo más probable es que la rapaz que acumulo los restos de roedores encontrados viviese muy cerca de la zona de depósito final, posiblemente en alguna repisa situada en alguno de los extremos de la sima.

\section{RESULTADOS}

Los taxones presentes en Kiputz IX han sido identificados con anterioridad en otros yacimientos del Pleistoceno de la cornisa cantábrica, aunque su representación numérica varía dependiendo de la cronología (Fig. 4). Mientras que

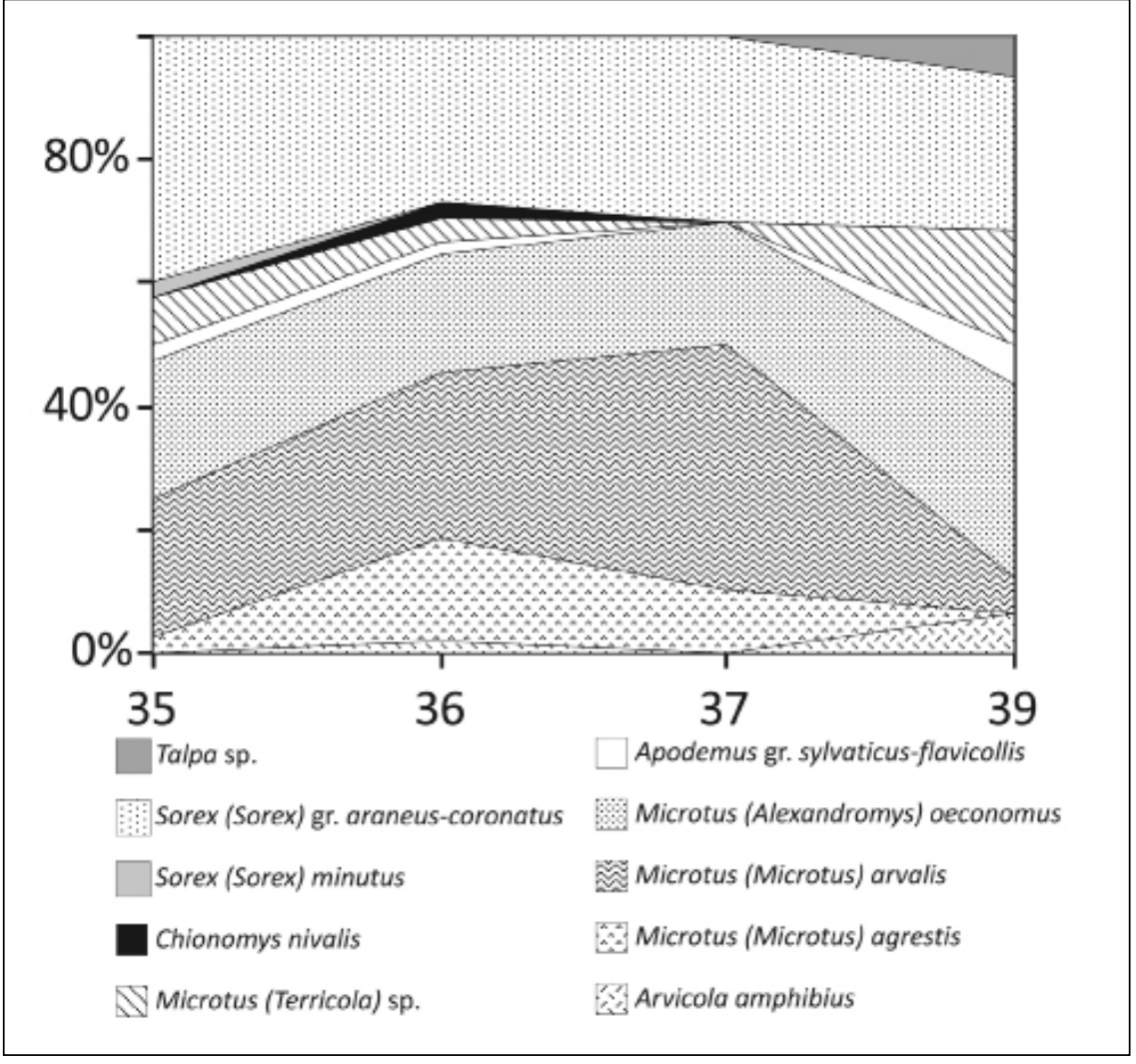

A Figura 4. Variación cuantitativa de los taxones (según el NMI) de las tallas coetáneas al Solutrense en el yacimiento de Kiputz IX (Mutriku, Gipuzkoa, España). 
Microtus (Microtus) agrestis, Microtus (Microtus) arvalis, Microtus (Alexandromys) oeconomus y Sorex gr. araneuscoronatus están presentes en todas las tallas estudiadas, hay otras especies que solo están presentes en una única talla, como es el caso de Chionomys nivalis, Sorex minutus y Talpa sp.

Es notoria la superioridad que tienen las especies indicativas de humedad, mientras que las especies relacionadas con climas menos húmedos y más templados como los géneros Clethrionomys, Eliomys o Glis están ausentes de esta secuencia. Por su parte, la presencia de taxones relacionados con medios de bosque y matorral es muy reducida, contando únicamente con 3 individuos entre los 114 identificados.

El volumen de sedimento recogido en cada talla ha sido similar, por lo que las variaciones en la riqueza de las muestras no están condicionadas por la cantidad de muestra obtenida. De esta manera, se observa que el número mínimo de individuos aumenta considerablemente en las dos tallas superiores, ya que pasamos de un mínimo de 16 y 10 individuos en las tallas 39 y 37 respectivamente a tener 48 y 40 en las tallas 36 y 35 . Otras variaciones entre las dos tallas inferiores y las superiores también son considerables. Si tenemos en cuenta la diversidad, es la talla 37 la que presenta los valores más bajos (1.28 obtenido con la fórmula de Shannon Wiener) mientras que las otras tres tallas presentan valores similares (1.7 para la talla $39 ; 1.6$ para la 36 y 1.5 para la 35). Coincide también que la talla 37 es en la que se han identificado el menor número de taxones (cuatro) y de hábitat (tres).

Tanto en la base como en el techo de la secuencia solutrense se ve como la proporción relativa de las especies de pradera húmeda aumenta, mientras que las tallas 37 y 36 constituyen el momento de mayor expansión de las especies de pradera. Las especies de ribera están presentes en toda la secuencia, aunque no muy representadas. Aun así, aumentan cuando se incrementan también los otros biotopos indicadores de humedad. En lo que respecta a los biotopos de montaña y de bosque, su presencia en este yacimiento es muy reducida, el primero probablemente por no ser el entorno del yacimiento de elevada altitud y el segundo por ser un momento climáticamente más riguroso.

\section{CONCLUSIONES}

A partir de esta información, la reconstrucción paleoambiental del entorno de Kiputz IX (Fig. 5) en el momento de la formación de este depósito seria de una zona de praderas húmedas y con algún tipo de masa de agua constante en la cercanía en un momento climáticamente más húmedo y frio que el actual.

Este trabajo supone una primera aproximación a las tallas equivalentes a una edad cultural Solutrense del ya-

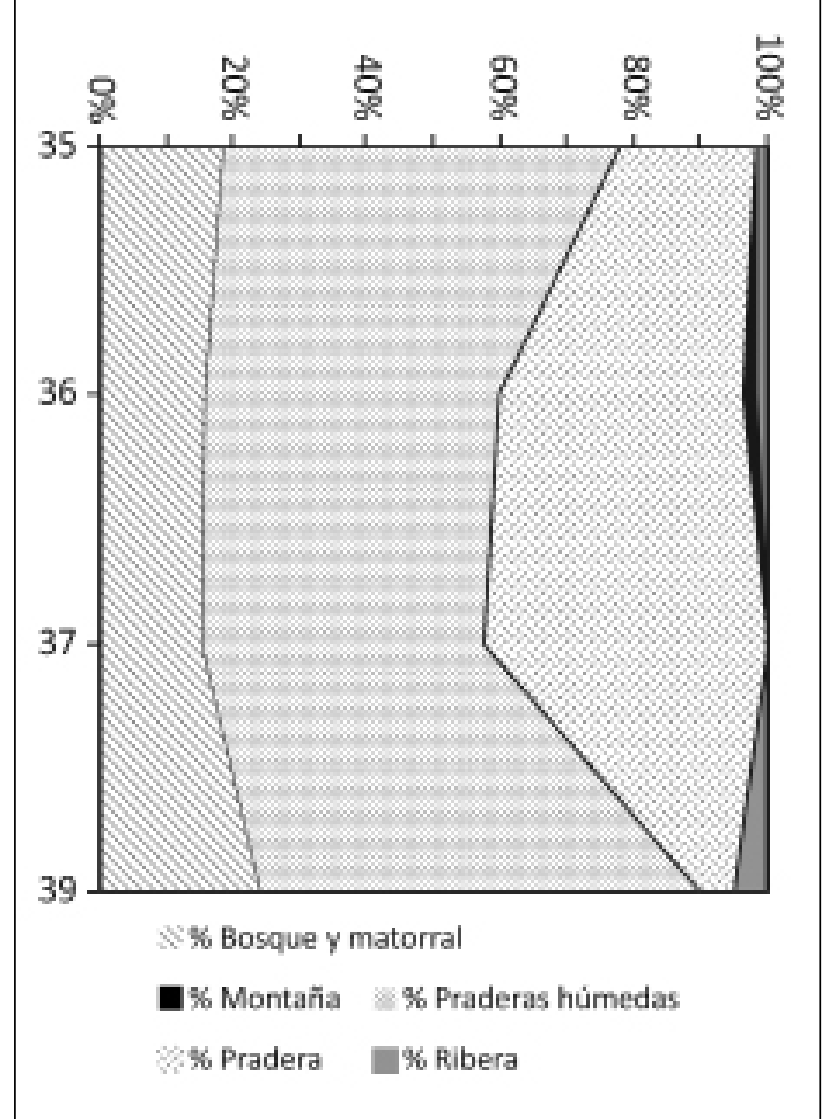

$\Delta$ Figura 5. Reconstrucción ecológica de las tallas 35, 36, $37 \mathrm{y}$ 39 del yacimiento de Kiputz IX (Mutriku, Gipuzkoa, España).

cimiento paleontológico de Kiputz IX. Es importante destacar que la visión ofrecida por este yacimiento no está distorsionada por actividades humanas, lo cual ofrece una mejor aproximación a la reconstrucción paleoambiental y paleoecológica.

De los 10 taxones identificados, todos excepto Apodemus sylvaticus-flavicollis son especies más abundantes en zonas de clima más húmedo y frio e indicadoras de humedad, lo cual responde a las estimaciones del momento cronológico en el que nos encontramos.

\section{AGRADECIMIENTOS}

Todos los estudios paleontológicos que se han realizado y se realizaran en el futuro son gracias al trabajo de prospección que ha realizado el Grupo Munibe de Azkoitia y más concretamente Miguel Sasieta y Juan M. ${ }^{a}$ Arruabarrena. Este trabajo ha sido financiado por la Diputación de Gipuzkoa y los proyectos Unesco 09/01, EHU08/84, EHU10/32 y GUI12/35 de la Universidad del País Vasco / Euskal Herriko Unibertsitatea. Uno de los autores (N.G-I.) disfruta de una beca de formación de investigadores (BFI-2010-289) del Gobierno Vasco. • 


\section{BIBLIOGRAFÍA}

AltunA, J. 1972: "Fauna de los mamíferos de los yacimientos de Guipúzcoa. Con Catálogo de los Mamíferos cuaternarios del Cantábrico y del Pirineo Occidental". Munibe 24: 1-464.

- 1980: "Hallazgo de un lince nórdico (Lynx lynx L. Mammalia) en la sima de Pagolusieta, Gorbea (Vizcaya)". Munibe 32 (3-4): 317-322.

AndRews, P. 1990: Owls, Caves and Fossils. Natural History Museum Publications, London.

Blanco, J.C. 1998: Mamíferos de España. II Tomos. Planeta S.A. Barcelona.

BRONK RAMSEY, C. 2009: "Bayesian analysis of radiocarbon dates". Radiocarbon 51: 337-360.

Castaños, P. M. 1996: "Hallazgo de rinoceronte lanudo en Legintxiki (Etxauri, Navarra)" Príncipe de Viana, Suplemento de Ciencias 1415: 77-80.

Castaños, P. 2005: "Cueva de Kiputz IX (Mutriku). I Campaña". Areoikuska 2004. Servicio Central de Publicaciones del Gobierno Vasco. Vitoria-Gasteiz: 141-143.

- 2006: "Cueva de Kiputz IX (Mutriku). II Campaña". Arkeoikuska 2005. Servicio Central de Publicaciones del Gobierno Vasco. VitoriaGasteiz: 136-138.

- 2007: "Cueva de Kiputz IX (Mutriku). III Campaña". Arkeoikuska 2006. Servicio Central de Publicaciones del Gobierno Vasco. VitoriaGasteiz: 190-192.

- 2008: "Cueva de Kiputz IX (Mutriku). IV Campaña". Arkeoikuska 2007. Servicio Central de Publicaciones del Gobierno Vasco. VitoriaGasteiz: 376-378.

Castaños, J., Castaños, P., Murelaga, X. y Alonso-Olazabal, A. 2012: "Kiputz IX: Un conjunto singular de bisonte estepario (Bison priscus, Bojanus, 1827) del Pleistoceno superior de la Península Ibérica". Ameghiniana 49 (2): 247-261.

Castaños, J., Castaños, P., Murelaga, X., Alonso-Olazabal, A., Ortega, L. A. y ZULuAGA, M. ${ }^{a}$ C. en prensa: "Osteometric analysis of scapula and humerus for Rangifer tarandus and Cervus elaphus: A contribution to cervid discrimination (Late Pleistocene, Southern Pyrenees)". Acta Palaeontologica Polonica, doi: http://dx.doi.org/10.4202/ app.2012.0027.

Corchón Rodríguez, M. S. 1990: "La Cueva de Las Caldas (Priorio, Oviedo). Investigaciones efectuadas entre 1980 y 1986". Excavaciones arqueológicas en Asturias, 1983-1986. Servicio de Publicaciones del Principado de Asturias. Oviedo: 37-53.

Cuenca-Bescós, G., Strauss, L. G., González Morales, M. R. y García PlMIENTA, J. C. 2008: "Paleoclima y paisaje del final del Cuaternario en Cantabria: Los pequeños mamíferos de la cueva del Mirón (Ramales de la Victoria)". Revista Española de Paleontología 23 (1): 91-126.

Cuenca-Bescós, G., Strauss, L. G., González Morales, M. R. y García PlMIENTA, J.C. 2009: "The reconstruction of past environments through small mammals: from the Mousterian to the Bronze Age in El Mirón Cave (Cantabria, Spain)". Journal of Archaeological Science 36: 947955.

Cuenca-Bescós, G. y García PimientA, J. C. 2012: "Holocene biostratigraphy and climatic change in Cantabria: the micromammalian faunas of El Mirón Cave (Ramales de la Victoria, Cantabria, Spain)". En L.G. Strauss y M.R. González Morales (eds.): El Mirón Cave, Cantabrian Spain. University of New Mexico Press. Albuquerque: 205-242.

EdESO Fito, J. M. 1993: "Itinerario N5: Valle de Lastur". Lurralde: Investigación y espacio 16: 195-214.

FAtı, V. 1867: Les campagnols du bassin du Léman. Association zoologique du Léman / 3. Bâle \& Genève.

FISCHER, G. 1814: Zoognosia tabulis synopticis illustrate: in usum praelectionum Academiae imperialis medico-chirugicae mosquensis 3. Nicolai Sergeidis Vsevolozsky, Moscow.

- 1817: "Adversaria Zoologica". Societatis Imperialis Naturae 5: 357428.
HEINRICH, W-D. 1978: "Zur biometrischen Erfassung eines Evolutionstrends bei Arvicola (Rodentia, Mammalia) aus dem Pleistozän Thüringens". Saugetierkundliche Informationen 2: 3-21.

- 1982: "Ein Evolutionstrend bei Arvicola (Rodentia, Mammalia) und seine Bedeutung für die Biostratigraphie im Pleistozän Europas". Wissenschaftliche Zeitschrift der Humboldt-Universität zu Berlin. Mathematisch-Naturwissenschaftliche Reihe XXXI (3): $155-160$.

ILLIGER, J. K. W. 1815: "Ueberbliok der Saügthiere nach ihrer Vertheilung über die Welttheile". Abhandlungen der physikalischen Klasse der Königlich-Preussischen Akademie der Wissenschaften 1804-1811: 39-160.

Kalthoff, D. C., Mörs, T. y TeSAKOV, A. 2007: "Late Pleistocene small mammals from the Wannenköpfe volcanoes (Neuwied basin, western Germany) with remarks on the stratigraphic range of Arvicola terrestris". Geobios 40: 609-623.

KAUP, J. J. 1829: Skizzierte Entwicklungsgeschichte und Natürliches System der Europäischen Tierwelt. Editor Carl Wilhelm Leske.

LACĖPÉDE, B. G. E. 1799: Tableau des divisions, sous-divisions, ordres et genres des mammifères. Plassan. Paris.

LINNAEUS, C. 1758: Systema Naturae per regna tria naturae, secundum classes, ordines, genera, species cum characteribus, differentiis, synonymis, locis. 1st Edition. Laurentii Salvii. Stockholmiæ.

- 1761: Fauna Svecica sistens animalia Svecia Regni: mammalia, aves, amphibia, pisces, insecta, vermes. Distributa per classes \& ordines, genera \& species, cum differentiis specierum, synonymis auctorum, nominibus incolarum, locis natalium, descriptionibus insectorum. Sumtu \& Literis Direct. Laurentii Salvii. Stockholmiæ.

- 1766-1768: Systema naturae per regna tria naturae, secundum classes, ordines, genera, species, cum characteribus, differentiis synonymis, locis. 12th Edition. Laurentii Salvii. Stockholm.

López-García, J. M., Blain, H-A., De Marfà I Taillefer, R., García, A., MartiNelL, J., Bennasar Félix, M. L. y Cuenca-Bescós, G. 2011: "Small-mammals from the Middle Pleistocene layers of the Sima del Elefante (Sierra de Atapuerca, Burgos, northwestern Spain)". Geological Acta 9: 29-43.

López-García, J. M., Blain, H-A., Molares, J.I., Lorenzo, C., Bañuls-CarDONA, S. y CUENCA-BESCÓS, G. 2013: "Small-mammal diversity in Spain during the late Pleistocene to early Holocene: Climate, landscape and human impact". Geology 41 (2): 267-270.

MARTINS C. 1842: "Note sur I' Arvicola nivalis, nouvelle espèce de campagnol habitant la région des neiges éternelles dans les Alpes de la Suisse". Annales des sciences naturelles. Zoologie 2: 87-100.

MERLCHIOR. 1834: Dansk. Staar. Norg. Patterdyr: 99.

Miller, G. S. 1908: "Eighteen new European voles". Annals and Magazine of Natural History 8 (1): 194-206.

OGNEV, S.I. 1914: "Mammals of lower reaches of the river Tuman-Gana (southern part of Primorsk region). Pt. I. Rodentia". Dnevnik Zool. Otd. Ob-va Lyubitelei Estestvoznaniya, Antropologii i Etnografii N. Society 2 (3): 101-128.

PALLAS, P. S. 1776: Reise durch verschiedene Provinzen des Russischen Reichs. Academie der Biffenfchaften. St. Petersbourg.

PALOMO, L. J. y GISBERT, J. 2002: Atlas de Mamíferos Terrestres de España. Organismo Autónomo de Parques Nacionales. DGNA-MIMAM. Madrid.

PEMÁN, E. 1985: "Aspectos climáticos y ecológicos de los micromamíferos del yacimiento de Erralla". Munibe 37: 49-57.

- 1989: "Los micromamíferos de la Cueva de Amalda y su significado. Comentarios sobre Pliomys lenki (Heller, 1930) (Rodentia, Mammalia)". En J. Altuna; A. Baldeón y K. Mariezkurrena (Dir.): La cueva de Amalda (Zestoa, País Vasco): ocupaciones paleolíticas y postpaleolíticas. Eusko Ikaskuntza. Donostia: 225-238.

- 1994: "Los micromamíferos de Lamiñak II (Berriatua, Bizkaia)". Kobie XXI: 225-233. 
PoKINES, J. T. 1998: The Paleoecology of Lower Magdalenian Cantabrian Spain. BAR International Series, 713. Oxford, British Archaeological Reports.

Reimer, P. J., Baillie, M. G., Bard, E., Bayliss, A., Beck, J. W., Blackwell, P. G., Bronk Ramsey, C., Buck, C. E., BurR, G., EdWards, R. L., Friedrich, M., Grootes, P., Guilderson, T., Hajdas, I., Heaton, T. J., Hogg, A. G., Hughen, K. A., Kaiser, K. F., Kromer, B., Mccormac, E. G., Manning, S. W., Reimer, S. D., Richards, D. A., Southon, J., Talamo, S., Turney, C., Van Der Plicht, J. y WeYhenmeYer, C. E. 2009: "Intcal09 and Marine09 radiocarbon age calibration curves, 0-50 000 years cal BP." Radiocarbon 51 (3): 1111-1150.

REUMER, J. W. F. 1984: "Ruscinian and early Pleistocene Soricidae (Insectivora, Mammalia) from Tegelen (The Netherlands) and Hungary". Scripta Geologica 73: 1-173.

Rofes, J., Bover, P., Cuenca-Bescós, G. y AlCover, J. A. 2012: "Nesiotites refelinensis sp. nov., the earliest shrew (Mammalia, Soricidae) from the Balearic Islands, Spain". Palaeontologia Electronica 15 (1): 8A, 12 p.

SCHRANK, F. P. 1798: Fauna Boica. Durchgedachte Gefchichte der in Baiern einheimifchen und zahmen Thiere. Stein'schen Buchhandlung. Germany.
SESÉ, C. 2005: "Aportación de los micromamíferos al conocimiento paleoambiental del Pleistoceno. Nuevos datos y sintesis Superior en la Región Cantábrica". Monografías del Centro de Investigación y Museo de Altamira 20: 167-200.

Straus, L.G. y Clarck, G. A. 1986: La Riera Cave. Stone Age HunterGatherer Adaptations in Northern Spain. Anthropological Research Papers, 36. Arizona.

Van Der Meulen, A. 1973: "Middle Pleistocene smaller Mammals from the Monte Peglia (Orviedo, Italy), with Special Reference to the Phylogeny of Microtus (Arvicolidae, Rodentia)". Quaternaria 17: 1-144.

Villaluenga, A. 2011: "Sima de Illobi (Aralar, Navarra) yacimiento de oso pardo (U. arctos Linnaeus, 1758). Estudio taxonómico y tafonómico". Munibe 62: 145-174.

Waddell, P. J., OKAdA, N. y HaSegawa, M. 1999: "Towards resolving the interordinal relationships of placental mammals". Systematic Biology 48: 1-15.

Wilson, D. E. y Reeder, D. M. 2005: Mammal Species of the World. A Taxonomic and Geographic Reference. John Hopkins University Press. Baltimore. 
